\title{
Hierarchy of One-Dimensional Models in Nonlinear Elasticity
}

\author{
JEAN-JACQUES MARIGO ${ }^{1, \star}$ and NICOLAS MEUNIER ${ }^{2}$ \\ ${ }^{1}$ Laboratoire de Modélisation en Mécanique (UMR 7607), Université Pierre et Marie Curie, \\ 4 Place Jussieu, 75252, Paris cedex 05, France.E-mail: marigo@Imm.jussieu.fr \\ ${ }^{2}$ Laboratoire Jacques-Louis Lions (UMR 7598), Université Pierre et Marie Curie, \\ 175 rue du Chevaleret, Paris 75013, France.E-mail: meunier@ann.jussieu.fr
}

Received 18 April 2005; in revised form 24 September 2005

\begin{abstract}
By using formal asymptotic expansions, we build one-dimensional models for slender hyperelastic cylinders submitted to conservative loads. According to the order of magnitude of the applied loads, we obtain a hierarchy of models going from the linear theory of flexible bars to the nonlinear theory of extensible strings.
\end{abstract}

Résumé. On construit, à l'aide de développements asymptotiques formels, des modèles unidimensionnels de cylindres hyperélastiques élancés soumis à des forces conservatives. Suivant l'ordre de grandeur des forces appliquées, on obtient une hiérarchie de modèles allant de la théorie des poutres flexibles jusqu'à la théorie des fils élastiques.

Mathematics Subject Classifications (2000): 74K10, 74B05, 74G65, 74G10, 35A15, 35C20.

Key words: elasticity, rods, strings, asymptotic expansions, variational methods.

\section{Introduction}

Engineers use various one-dimensional models to describe the elastostatic response of slender three-dimensional structures subjected to conservative loads. The most used are the theory of elastic strings, the theory of inextensible strings, the nonlinear theory of elastic straight beams and the linear theory of elastic straight beams, see $[2,20,34]$. The way of choosing the model seeming to be the most appropriate to the considered situation does not proceed of a thorough analysis and remains very intuitive, because a hierarchy between these various models is not clearly established. Furthermore, the question of knowing whether this list of models is comprehensive, or whether there is not any intermediate model, remains unanswered. The only way to answer would be to follow a deductive process in order to construct one-dimensional models starting from three-dimensional elasticity.

\footnotetext{
$\star$ Corresponding author.
} 
Unfortunately, the first concern of the scientific researchers has been to separately justify each existing model by introducing appropriate hypotheses, in general explicitly or implicitly connected to specific choices of the scaling of the applied forces. These justifications, which start from the three-dimensional theory, are made by using either formal asymptotic methods such as the method of asymptotic expansions or rigorous mathematical arguments such as $\Gamma$-convergence techniques. For further informations, the reader could refer to [35] for a broad review of the bibliography (up to 1996). Let us give a brief overview of those most important justifications. Starting with the work of [31] for beams and the works of [8] for plates, a first complete asymptotic analysis of linearly elastic straight beams based on formal asymptotic expansions can be found in [6]. [14] give a rigorous proof of convergence (based on classical tools of functional analysis) in the context of partially anisotropic, heterogeneous and linearly elastic straight beams. That work was first extended by [15], then by [32] to full anisotropic and heterogeneous beams. In the framework of nonlinear elasticity, [9] provides the first attempt to justify nonlinear models of elastic straight beams by formal asymptotic methods. A derivation of a nonlinear bending-torsion model for inextensible rods by $\Gamma$-convergence was proposed by [25]. The models of extensible or inextensible strings have been justified by rigorous mathematical arguments by [1] or [26]. There is also a great number of works devoted to the justification of various models of membranes, plates or shells associated to thin structures, see for instance [7] and [12].

Very few works have been devoted to a hierarchical organization of these models in order to help the engineers make the right choice. To our knowledge, the work by [21] was the first in which such a hierarchy of rod models appeared. Unfortunately the details of the proofs were never published. Since this publication, a few works have been completed with the same objective of hierarchization. Let us particularly quote [13] which obtain by $\Gamma$-convergence a hierarchy of plate models. There are also the works by [23] and [24] in which, following the work by [29], is also obtained, formally, a hierarchy of rod models completely similar to the hierarchy introduced by [21]. The approach in [23, 24] is based on the resolution of a sequence of recursive minimization problems and presents the advantage of admitting a larger set of admissible deformation, thus removing an unnecessary hypothesis of [12] and obtaining an expression for the string energy that agrees with that found via $\Gamma$-convergence. The goal of the present paper is to detail the results announced by [21].

The main assumptions of our analysis are as follows: (i) the body is homogeneous, elastic and isotropic; (ii) its natural reference configuration is a cylinder; (iii) the applied forces are conservative (but not necessarily dead loads); (iv) the displacements are not necessarily infinitesimal and the elastic potential is non-linear. In this context, as it has been announced by [21], we obtain a hierarchy of four asymptotic one-dimensional models depending on the order of magnitude of the applied loads. 
To do so, we first introduce two dimensionless parameters, namely $\epsilon$ and $\eta$. The parameter $\epsilon$ is the traditional parameter of slenderness, i.e., the ratio between the cross-section diameter and the length of the cylinder. The parameter $\eta$ is the ratio between a parameter characteristic of the intensity of the applied forces and a parameter representative of the cylinder rigidity (in practice the product of the Young modulus of the material by the area of the cross-section). Then the parameter $\eta$ is compared with the small parameter $\epsilon$. Finally, denoting by $n$ the order of magnitude of the dimensionless load parameter $\eta$ compared to the slender parameter $\epsilon, \eta \sim \epsilon^{n}$, we obtain the following different models

1. When $n \geq 3$, the slender elastic cylinder behaves like a linear inextensible, flexible beam. The displacements are infinitesimal (of order $n-2$ ), the strain energy and the potential of the external forces are infinitesimal and both of order $2 n-2$. The leading term in the strain energy corresponds to the bending energy of inextensible infinitesimal Navier-Bernoulli displacements;

2. When $n=2$, we obtain the nonlinear model of an inextensible, flexible bar. The displacements are finite, but the strains are infinitesimal (of order 1), the strain energy and the potential of the external forces are both of order 2. The leading term of the strain energy corresponds to the bending energy (eventually coupled with a torsion energy) of inextensible finite NavierBernoulli displacements;

3. When $n=1$, the response of the slender elastic cylinder is similar to that of an inextensible string. The displacements are finite and inextensional. The potential of the external forces is of order 0 while the strain energy is negligible;

4. Finally, when $n=0$, we have to find the stable equilibrium of an elastic string. The displacements are finite, the potential of the external forces and the strain energy are of order 0 . The leading term of the strain energy corresponds to an extensional energy, the bending energy is negligible.

Thus, we see that these models are in conformity with those used by the engineers. Moreover, they reveal two great families of models: elastically flexible beams on the one hand, and perfectly flexible strings on the other hand. However, the originality of the present work, compared to what one can find in the 'classical' literature is that these models are associated with levels of intensity of the loading. Contrary to the daily experiment, the concept of a string or of a beam is not an intrinsic quality to a given slender body, but a 'type of behavior' induced by the intensity of the loads. The same object (made of the same material and with the same geometry) will behave as a flexible beam if the intensity of the loading is sufficiently weak and as an extensible string if the loading is rather strong. In the everyday life, what misleads our senses is that the objects of use are always subjected to the same type of loading and thus the same type of behavior is always observed. 
All these results are obtained by using techniques of formal asymptotic expansions and consequently they could appear to be less interesting than those obtained by $\Gamma$-convergence. However, apart from the postulate that the solution can be expended in powers of $\epsilon$, all the procedure is rigorous and deductive. Compared to the techniques of $\Gamma$-convergence, this method provides a construction process, in the sense that the orders of magnitude of the different energies and the shape of the optimal displacement fields are built 'gradually' during the estimate process. Moreover the analysis is strongly based on Hypothesis 3.3 relating to the type of applied forces. Roughly speaking this condition requires that the applied forces produce work in inextensional displacements of the Navier-Bernoulli type. This assumption guarantees that each model obtained is not degenerated, in other words that the leading term of the energy is genuinely not vanishing. Here, one can still regret that all the works of justification based on the asymptotic methods overshadow in a quasi-systematic way this question that however always arises. Should the condition not be satisfied, the model thus obtained does not apply any more and the analysis should be refined. This question reminds the issue of the completeness of the models list. We will not treat it in this article and shall reserve it for future publications. The reader interested in this question should consult the work of [22] to have an idea of the extent of the task. What clearly appears is that the number of asymptotic models can vary ad infinitum if one exploits the various parameters relating to the loading, the geometry or the behavior. What one can hope for is that the method used here is rather flexible and general enough to be adapted to any situation.

Specifically, the paper is organized as follows: In Section 2, we fix the notations and the context of the work. In Section 3, we introduce the ingredients of the asymptotic analysis. Section 4 is devoted to the construction of the different types of displacements and to the classification of the energies which will appear in the asymptotic models. In Section 5 we obtain the right order of magnitude of the displacements and of the energies in relation with the order of magnitude of the loading. Section 6 is devoted to the presentation of the hierarchy of one-dimensional models so obtained. The long proof of Theorem 4.1 is reported to an appendix. In general, the intrinsic notation is preferred to the component notation. However, when the component notation is chosen, we use the summation convention on repeated indices. Latin indices $\mathrm{i}, \mathrm{j}, \mathrm{k}, \ldots$ take their value in the set $\{1,2,3\}$ while Greek indices $\alpha, \beta, \gamma, \ldots$ (except $\epsilon$ ) in the set $\{1,2\}$. Moreover, we denote by $u,{ }_{i}$ the derivative of $u$ with respect to $x_{i}$, the components of the vector $u$ are denoted $u_{i}$. The group of the rotations is denoted $S O(3)$ and the linear space of skew-symmetric $3 \times 3$ matrices is denoted $S k(3)$.

\section{The Three-dimensional Problem}

Let $\hat{\omega}$ be an open, bounded and connected subset in $\mathbb{R}^{2}$ with diameter R. Given $\mathrm{L}>0$, we denote by $\hat{\Omega}$ the cylinder $\hat{\Omega}=\hat{\omega} \times(0, \mathrm{~L})$ the generic point of which is 
denoted by $\hat{x}=\left(\hat{x}_{1}, \hat{x}_{2}, \hat{x}_{3}\right)$. The volume element of $\hat{\Omega}$ is denoted $\mathrm{d} \hat{x}=\mathrm{d} \hat{x}_{1} \mathrm{~d} \hat{x}_{2} \mathrm{~d} \hat{x}_{3}$ and the gradient with respect to $\hat{x}$ is denoted by $\hat{\nabla}$. We assume that the section $\hat{\Gamma}_{0}=\hat{\omega} \times\{0\}$ is clamped - the role of this condition is simply to rule out any rigid displacement - while everywhere else, the body is submitted to a system of body or surface conservative forces which are supposed to derive from the potential $\hat{\mathcal{L}}$. Thus, the set $\hat{\mathcal{C}}$ of admissible displacement fields reads as

$$
\hat{\mathcal{C}}=\left\{\hat{v}: \hat{v} \text { “smooth", }\left.\hat{v}\right|_{\hat{\Gamma}_{0}}=0, \operatorname{det}(\hat{\nabla} \hat{v}+I)>0\right\} .
$$

In (1) the condition det $\hat{F}>0$, with $\hat{F}=I+\hat{\nabla} \hat{v}$, ensures that the deformation preserves the orientation and it is unnecessary to precise the regularity of the fields in our formal approach. The Green-Lagrange strain tensor $\hat{E}$ is given by $2 \hat{E}=\hat{F}^{T} \hat{F}-I$.

Let us note that, contrary to usual assumptions made by [12, 29] or [23], we do not suppose that the loads are dead loads. Thus, the potential of the external forces $\hat{\mathcal{L}}$ is not necessarily a linear form on $\hat{\mathcal{C}}$, but enjoys the following properties:

1. $\hat{\mathcal{L}}$ is a smooth function defined on $\hat{\mathcal{C}}$, which vanishes when the body is in its reference configuration, $\hat{\mathcal{L}}(0)=0$.

2. The derivative of $\hat{\mathcal{L}}$ at $0, \hat{\mathcal{L}}^{\prime}(0)$, is a non-vanishing continuous linear form on the set of displacements of $H^{1}\left(\hat{\Omega} ; \mathbb{R}^{3}\right)$ satisfying the clamping condition. Its norm $F=\left\|\hat{\mathcal{L}}^{\prime}(0)\right\|$ will be used to define the order of magnitude of the loading.

The domain $\hat{\Omega}$ is the natural reference configuration of an elastic isotropic homogeneous body. The elastic potential $\hat{W}$ enjoys the following properties:

1. $\hat{W}$ is a smooth, isotropic, non-negative function that only depends on $\hat{E}$.

2. $\hat{W}$ vanishes only at $\hat{E}=0$, and near $\hat{E}=0$ it admits the following development

$$
2 \hat{W}(\hat{E})=\lambda \operatorname{tr}(\hat{E})^{2}+2 \mu \hat{E} \cdot \hat{E}+o(\hat{E} \cdot \hat{E}),
$$

where the Lamé coefficients $\lambda$ and $\mu$ are related to Young's modulus $\mathbf{E}$ and Poisson's ratio $\nu$ via the classical formulae

$$
\mathrm{E}=\frac{\mu(3 \lambda+2 \mu)}{\lambda+\mu}, \quad \nu=\frac{\lambda}{2(\lambda+\mu)} .
$$

These elastic coefficients satisfy the usual inequalities

$$
\mathrm{E}>0, \quad-1<\nu<\frac{1}{2}, \quad \mu>0, \quad 3 \lambda+2 \mu>0 .
$$


3. $\hat{W}$ satisfies the following growth condition near infinity:

$$
\hat{W}(\hat{E}) \geq a\|\hat{E}\|^{p}+b, \text { with } a>0 \text { and } 2 p>1 \text {, when }\|\hat{E}\| \rightarrow+\infty .
$$

4. $\hat{W}$ satisfies the 'right' poly or quasi convexity together with the coercivity conditions, see [4] or [27].

The research of stable equilibrium configuration of this body leads to the minimization of the potential energy, which is the difference between the strain energy and the potential of the external loads. So, the problem reads as

Find $\hat{u} \in \hat{\mathcal{C}}$ minimizing $\hat{\mathcal{P}}(\hat{v})$ for $\hat{v} \in \hat{\mathcal{C}} \quad$ with $\hat{\mathcal{P}}(\hat{v})=\int_{\hat{\Omega}} \hat{W}(\hat{E}(\hat{v}(\hat{x}))) \mathrm{d} \hat{x}-\hat{\mathcal{L}}(\hat{v})$.

REMARK 2.1. We assume that all the previous conditions are such that this minimization problem admits (at least) one solution, see [4, 5, 11, 27] for more precise statements.

\section{Setting of the Asymptotic Procedure}

We will assume that the body is slender in the sense that its natural length $L$ can be considered large in comparison to $R$. Therefore, we introduce the slenderness parameter

$$
\epsilon=\frac{\mathrm{R}}{\mathrm{L}}
$$

and consider that it is small with respect to 1 .

We intend to study the behavior of the sequence of the displacements of the body at equilibrium as $\epsilon$ goes to zero by the asymptotic procedure summarized below. The key steps in the analysis are: (i) A rescaling which transports the problem to a domain $\Omega$ that does not depend on $\epsilon$; (ii) An asymptotic expansion of the rescaled loads and equilibrium displacements in power of the small parameter $\epsilon$; (iii) The computation of the asymptotic expansion of the GreenLagrange tensor and of the energies. Each of these steps is described in details below. We consider the dimensionless ratio

$$
\eta=\frac{\mathrm{F}}{\mathrm{ER}^{2}}
$$

as the parameter characterizing the relative intensity of the loading. The loading parameter $\eta$ must be compared to the slenderness parameter $\epsilon$ and we assume that

HYPOTHESIS 3.1. There exists $\bar{\eta}>0$ and $n \in \mathbb{N}$ such that $\eta=\bar{\eta} \epsilon^{n}$. 


\subsection{THE THREE-DIMENSIONAL RESCALED PROBLEMS}

For each $\epsilon>0$, the displacement $\hat{v}$ of the body in the equilibrium configuration is defined on an open set $\hat{\Omega}$ which depends on $\epsilon$. In order to perform an asymptotic analysis, it is useful to consider an open set which is independent of $\epsilon$. We adopt the following straightforward change of coordinates, in the fashion introduced by [8]. We associate to any displacement field defined on $\hat{\Omega}$ a dimensionless displacement field $v$ defined on $\Omega=\omega \times(0,1)$ by:

$$
\hat{v}(\hat{x})=\operatorname{L} v(x), \text { with } x=\left(\hat{x}_{1} / \mathrm{R}, \hat{x}_{2} / \mathrm{R}, \hat{x}_{3} / \mathrm{L}\right) .
$$

The clamped section becomes $\Gamma_{0}=\omega \times\{0\}$. The volume element of $\Omega$ is denoted $\mathrm{d} x=\mathrm{d} x_{1} \mathrm{~d} x_{2} \mathrm{~d} x_{3}$.

REMARK 3.1. In the sequel, dimensionless quantities associated with a given physical quantity are denoted without hat.

The research of the stable equilibrium configuration of the body in the new system of coordinates leads to the minimization problem parameterized by $\epsilon$, for given $n$ and $\bar{\eta}$ :

$$
\text { Find } u^{\epsilon} \text { minimizing } \mathcal{P}^{\epsilon}(v)=\int_{\Omega} W\left(E^{\epsilon}(v)\right) \mathrm{d} x-\bar{\eta} \epsilon^{n} \mathcal{L}^{\epsilon}(v) \text { for } v \in \mathcal{C}^{\epsilon} \text {. }
$$

In equation (6), $W(\cdot)=\hat{W}(\cdot) / \mathrm{E}$ and $\mathcal{L}^{\epsilon}(v)=\mathcal{L}^{\epsilon}(\hat{v}) / \mathrm{FL}$ are the dimensionless energies. The Green-Lagrange strain tensor associated to $v$ now reads

$$
2 E^{\epsilon}(v)=F^{\epsilon}(v)^{T} F^{\epsilon}(v)-I
$$

where $F^{\epsilon}(v)$ represents the dimensionless deformation gradient associated to $v$

$$
F^{\epsilon}(v)=I+\nabla_{\epsilon} v
$$

and $\nabla_{\epsilon}$ denotes the differential operator

$$
\nabla_{\epsilon}=\left(\frac{1}{\epsilon} \frac{\partial}{\partial x_{1}}\left|\frac{1}{\epsilon} \frac{\partial}{\partial x_{2}}\right| \frac{\partial}{\partial x_{3}}\right) .
$$

The set of admissible displacement fields associated with $\Omega$ is now defined by

$$
\mathcal{C}^{\epsilon}=\left\{v: \Omega \rightarrow \mathbb{R}^{3} \mid v \text { "smoth", }\left.v\right|_{\Gamma_{0}}=0, \operatorname{det}\left(\nabla_{\epsilon} v+I\right)>0\right\}
$$

and the total strain energy stored in the body is denoted $\mathcal{W}^{\epsilon}(v)$,

$$
\mathcal{W}^{\epsilon}(v)=\int_{\Omega} W\left(E^{\epsilon}(v)\right) \mathrm{d} x
$$


Near $E=0$, the dimensionless strain potential admits the following development:

$$
W(E)=\frac{1}{2} C E \cdot E+o(E \cdot E)
$$

where the fourth-order dimensionless rigidity tensor is given by

$$
C_{i j k l}=\frac{\nu}{(1+\nu)(1-2 \nu)} \delta_{i j} \delta_{k l}+\frac{1}{2(1+\nu)}\left(\delta_{i k} \delta_{j l}+\delta_{i l} \delta_{j k}\right) .
$$

\subsection{ASYMPTOTIC EXPANSION}

The parameter $\epsilon$ is assumed to be small with respect to 1 and will be used to define asymptotic expansions of the fields and the energies. Let us begin this section with some convention. Let $f^{\epsilon}$ be a function of $\epsilon$ which can be expanded in power of $\epsilon$, that is

$$
\exists q \in \mathbb{Z} \text { such that } f^{\epsilon}=\sum_{i \in \mathbb{Z}} \epsilon^{i} f^{i} \text { with } f^{i}=0 \forall i<q .
$$

We denote by $\mathcal{O}\left(f^{\epsilon}\right)$ the order of magnitude (called also the order) of $f^{\epsilon}$, the power of the first non-vanishing term in its expansion. By convention, the order is infinite when $f^{\epsilon}=0$. Therefore, if $f^{\epsilon} \neq 0$ can be expanded in power of $\epsilon$, we obtain:

$$
f^{\epsilon}=\sum_{i \in \mathbb{N}} \epsilon^{p+i} f^{p+i}, \mathcal{O}\left(f^{\epsilon}\right)=p \in \mathbb{Z}, f^{p} \neq 0 .
$$

In the three-dimensional rescaled problems, the solution $u^{\epsilon} \in \mathcal{C}^{\epsilon}$ and the load potential $\mathcal{L}^{\epsilon}$ depend parametrically on $\epsilon$. The next step of the asymptotic technique consists in assuming that the data of the problem admit an asymptotic expansion.

HYPOTHESIS 3.2. The potential of the applied forces $\mathcal{L}^{\epsilon}$ admits the following expansion in powers of $\epsilon$ :

$$
\mathcal{L}^{\epsilon}=\mathcal{L}^{0}+\epsilon \mathcal{L}^{1}+\epsilon^{2} \mathcal{L}^{2}+\ldots
$$

Moreover, the growth of $\mathcal{L}^{\epsilon}$ near infinity is at most linear: $\left|\mathcal{L}^{\epsilon}(v)\right| \leq a\|v\|+b$, when $\|v\| \rightarrow \infty$.

REMARK 3.2. Since by hypothesis $\mathcal{L}^{\epsilon}(0)=0$ we obtain that $\mathcal{L}^{i}(0)=0, \forall i \in \mathbb{N}$. The derivative of $\mathcal{L}^{0}$ at 0 is a linear form denoted by $\ell^{0}$ :

$$
\ell^{0}(v) \equiv \mathcal{L}^{0^{\prime}}(0)(v)
$$


Finally, we introduce an hypothesis that plays a fundamental role to obtain the right energy estimates. It will be called the non-degenerated loading condition.

HYPOTHESIS 3.3. Let $\mathcal{C}_{f l}$ be the set of admissible infinitesimal inextensional Navier-Bernoulli displacements,

$$
\mathcal{C}_{f l}=\left\{v: \Omega \rightarrow \mathbb{R}^{3} \mid v(x)=V\left(x_{3}\right),\left(V_{1}, V_{2}\right) \in H^{2}(0,1)^{2}, V_{3}=0, V(0)=V^{\prime}(0)=0\right\} .
$$

The applied forces produce work for at least one such displacement fields, i.e., the restriction of $\ell^{0}$ to $\mathcal{C}_{f l}$ is not 0 .

We now make a fundamental Ansatz concerning the solution of the problem $\mathcal{P}^{\epsilon}$. In terms of the displacement field it says:

ANSATZ 3.1. The displacement $u^{\epsilon}$ of the rescaled body in the equilibrium configuration can be expanded in powers of $\epsilon$ as

$$
u^{\epsilon}(x)=\sum_{i \in \mathbb{N}} \epsilon^{\mathcal{O}\left(u^{\epsilon}\right)+i} u^{\mathcal{O}\left(u^{\epsilon}\right)+i}, \mathcal{O}\left(u^{\epsilon}\right) \in \mathbb{Z}, u^{\mathcal{O}\left(u^{\epsilon}\right)} \neq 0 .
$$

REMARK 3.3. Since $F^{\epsilon}\left(u^{\epsilon}\right)=I+\frac{1}{\epsilon}\left(u_{, 1}^{\epsilon}\left|u_{, 2}^{\epsilon}\right| 0\right)+\left(0|0| u_{3}^{\epsilon}\right)$, then $F^{\epsilon}\left(u^{\epsilon}\right)$ admits an expansion in powers of $\epsilon$ and similarly $E^{\epsilon}\left(u^{\epsilon}\right)=\frac{1}{2}\left(F^{\epsilon T}\left(u^{\epsilon}\right) F^{\epsilon}\left(u^{\epsilon}\right)-I\right)$ does.

REMARK 3.4. Since $u^{\epsilon}$ is the displacement of the rescaled body in the equilibrium configuration, it minimizes $\mathcal{P}^{\epsilon}$, on the other hand, thanks to the clamping condition, $0 \in \mathcal{C}^{\epsilon}$, therefore,

$$
\mathcal{P}^{\epsilon}\left(u^{\epsilon}\right)=\mathcal{W}^{\epsilon}\left(u^{\epsilon}\right)-\bar{\eta} \epsilon^{n} \mathcal{L}^{\epsilon}\left(u^{\epsilon}\right) \leq \mathcal{P}^{\epsilon}(0)=0 .
$$

Using the hypothesis made on $\mathcal{L}^{\epsilon}$ together with the non-negative character of $W$, it follows that

$$
n+\mathcal{O}\left(u^{\epsilon}\right) \leq \mathcal{O}\left(\epsilon^{n} \mathcal{L}^{\epsilon}\left(u^{\epsilon}\right)\right) \leq \mathcal{O}\left(\mathcal{W}^{\epsilon}\left(u^{\epsilon}\right)\right) .
$$

PROPOSITION 3.1. The order of magnitude of the displacement is greater or equal to zero: $\mathcal{O}\left(u^{\epsilon}\right) \geq 0$.

Proof. (Notation: Throughout the proof one uses simplified notations, $F^{\epsilon}=$ $F^{\epsilon}\left(u^{\epsilon}\right)$ and $E^{\epsilon}=E^{\epsilon}\left(u^{\epsilon}\right)$.) Let us assume that $\mathcal{O}\left(u^{\epsilon}\right)=q<0$. Using the fact that $u^{\epsilon} \in \mathcal{C}^{\epsilon}$ together with the clamping condition, we obtain that $\left(u_{, 1}^{q}\left|u_{, 2}^{q}\right| u_{, 3}^{q}\right) \neq$ $(0|0| 0)$. Recalling that

$$
F^{\epsilon}=I+\frac{1}{\epsilon}\left(u_{, 1}^{\epsilon}\left|u_{, 2}^{\epsilon}\right| 0\right)+\left(0|0| u_{, 3}^{\epsilon}\right),
$$

we see that $q-1 \leq \mathcal{O}\left(F^{\epsilon}\right) \leq q<0$.

If $\left(u_{, 1}^{q} \mid u_{, 2}^{q}\right) \neq(0 \mid 0)$, then the first term of the expansion of $E^{\epsilon}$ is:

$$
E_{\alpha \beta}^{2 q-2}=\frac{1}{2} u_{, \alpha}^{q} \cdot u_{, \beta}^{q}, \quad E_{\alpha 3}^{2 q-2}=E_{33}^{2 q-2}=0 .
$$

Therefore, we deduce that $\mathcal{O}\left(E^{\epsilon}\right)=2 q-2<-2$. 
If $u_{\alpha}^{q}=0$, thanks to the clamping condition we deduce that $u_{3}^{q} \neq 0$. Then, we observe that $E_{i j}^{2 q-1}=0$ and $E_{33}^{2 q}=\frac{1}{2}\left|u_{3}^{q}\right|^{2} \neq 0$. So, in any case, $\mathcal{O}\left(E^{\epsilon}\right) \leq$ $2 \mathcal{O}\left(u^{\epsilon}\right)<0$.

Consequently, thanks to equations (3) and (16), it follows that

$$
n+\mathcal{O}\left(u^{\epsilon}\right) \leq \mathcal{O}\left(\epsilon^{n} \mathcal{L}^{\epsilon}\left(u^{\epsilon}\right)\right) \leq \mathcal{O}\left(\mathcal{W}^{\epsilon}\left(u^{\epsilon}\right)\right) \leq 2 p \mathcal{O}\left(u^{\epsilon}\right)<0,
$$

which is not possible.

Next, we give the expansion of the strain components. The following Lemma is obtained by expanding the displacement $u^{\epsilon}$ together with a direct computation.

LEMMA 3.2. The Green-Lagrange tensor $E^{\epsilon}\left(u^{\epsilon}\right)$ admits the following asymptotic expansion

$$
E^{\epsilon}\left(u^{\epsilon}\right)=\sum_{m=-2}^{\infty} E^{m}\left(u^{\epsilon}\right) \epsilon^{m},
$$

with

$$
\mathcal{O}\left(E_{\alpha \beta}^{\epsilon}\left(u^{\epsilon}\right)\right) \geq-2, \quad \mathcal{O}\left(E_{\alpha 3}^{\epsilon}\left(u^{\epsilon}\right)\right) \geq-1, \quad \mathcal{O}\left(E_{33}^{\epsilon}\left(u^{\epsilon}\right)\right) \geq 0 .
$$

More precisely, the components $E_{i j}^{m}$ have the following expression

$$
\begin{aligned}
& \forall m \geq-2, \quad 2 E_{\alpha \beta}^{m}\left(u^{\epsilon}\right)=\sum_{q=0}^{m+2} u_{, \alpha}^{q} \cdot u_{, \beta}^{m+2-q}+u_{\alpha, \beta}^{m+1}+u_{\beta, \alpha}^{m+1}, \\
& \forall m \geq-1, \quad 2 E_{\alpha 3}^{m}\left(u^{\epsilon}\right)=\sum_{q=0}^{m+1} u_{, \alpha}^{q} \cdot u_{, 3}^{m+1-q}+u_{\alpha, 3}^{m}+u_{3, \alpha}^{m+1}, \\
& \forall m \geq 0, \quad 2 E_{33}^{m}\left(u^{\epsilon}\right)=\sum_{q=0}^{m} u_{, 3}^{q} \cdot u_{, 3}^{m-q}+2 u_{3,3}^{m},
\end{aligned}
$$

with the convention that $u^{q}=0$ if $q<0$.

\section{Classification of the Displacements and of the Energies}

In this section, we study the relation between the different orders of magnitude of a displacement $v^{\epsilon}$ of $\mathcal{C}^{\epsilon}$ and its corresponding Green-Lagrange tensor $E^{\epsilon}\left(v^{\epsilon}\right)$. Since $\mathcal{O}\left(u^{\epsilon}\right) \geq 0$, it is enough to consider test functions $v^{\epsilon}$ which are such that 
$\mathcal{O}\left(v^{\epsilon}\right) \geq 0$. We distinguish the following types of displacement fields the name of which is justified by Theorem 4.1:

DEFINITION 4.1 (Displacements of order 0 without strain at negative order). We say that $v^{\epsilon} \in \mathcal{C}^{\epsilon}$ is of Class $\mathbf{0}$ if $\mathcal{O}\left(v^{\epsilon}\right)=0$ and

$$
v^{0}(x)=V\left(x_{3}\right) .
$$

DEFINITION 4.2 (Displacements of order 1 without strain at order 0 ). We say that $v^{\epsilon} \in \mathcal{C}^{\epsilon}$ is of Class 2 if $\mathcal{O}\left(v^{\epsilon}\right)=1$ and

$$
v^{1}(x)=\left(R\left(x_{3}\right)-I\right)\left(x-x_{3} e_{3}\right)+V\left(x_{3}\right),
$$

with $R\left(x_{3}\right) \in S O(3)$ and $R\left(x_{3}\right) e_{3}=e_{3}$.

DEFINITION 4.3 (Displacements of order $q, q \geq 2$, without strain at order $q-1)$. We say that $v^{\epsilon} \in \mathcal{C}^{\epsilon}$ is of Class 4 if $\mathcal{O}\left(v^{\epsilon}\right)=q, q \geq 2$, and

$$
v^{q}(x)=\theta_{v}\left(x_{3}\right) e_{3 \wedge} x+V\left(x_{3}\right) .
$$

DEFINITION 4.4 (Displacements of order 0 with strain at order 1). We say that $v^{\epsilon} \in \mathcal{C}^{\epsilon}$ is of Class 1 if $\mathcal{O}\left(v^{\epsilon}\right)=0$ and

$$
\begin{aligned}
& v^{0}(x)=V\left(x_{3}\right), \\
& v^{1}(x)=\left(R\left(x_{3}\right)-I\right)\left(x-x_{3} e_{3}\right)+\bar{V}\left(x_{3}\right),
\end{aligned}
$$

with $R\left(x_{3}\right) \in S O(3)$, and $V^{\prime}\left(x_{3}\right)=\left(R\left(x_{3}\right)-I\right) e_{3}$.

REMARK 4.1. Any displacement of Class $\mathbf{1}$ is a displacement of Class $\mathbf{0 .}$

DEFINITION 4.5 (Displacements of order $q, q \geq 1$, with strain at order $q+$ 1). We say that $v^{\epsilon} \in \mathcal{C}^{\epsilon}$ is of Class 3 if $\mathcal{O}\left(v^{\epsilon}\right)=q, q \geq 1$, and

$$
\begin{aligned}
v^{q}(x) & =V\left(x_{3}\right), \quad V_{3}=0, \\
v^{q+1}(x) & =\bar{\theta}_{v}\left(x_{3}\right) e_{3 \wedge} x-x \cdot V^{\prime}\left(x_{3}\right) e_{3}+\bar{V}\left(x_{3}\right) .
\end{aligned}
$$

REMARK 4.2. Any displacement of Class 3 with $q=1$ is a displacement of Class 2 with $R\left(x_{3}\right)=I$ and $V_{3}=0$. Any displacement of Class 3 with $q \geq 2$ is a displacement of Class 4 with $\theta_{v}=V_{3}=0$.

REMARK 4.3. For any displacement $v^{\epsilon}$ of Class 1 or 3 with $\mathcal{O}\left(v^{\epsilon}\right)=q$, the boundary condition at $x_{3}=0$ requires that $\left.v^{q}\right|_{x_{3}=0}=\left.v^{q+1}\right|_{x_{3}=0}=0$ and hence that $V(0)=V^{\prime}(0)=0$.

The following theorem is the main result of this section, it gives the orders of magnitude of the strain tensor in function of the order of the displacement field. For clarity we report its proof in the Appendix. 
THEOREM 4.1. Let $v^{\epsilon}$ in $\mathcal{C}^{\epsilon}$ and $E^{\epsilon}=E^{\epsilon}\left(v^{\epsilon}\right)$ its associated Green-Lagrange strain tensor. The following relations hold:

1. if $\mathcal{O}\left(v^{\epsilon}\right)=0$, then $-2 \leq \mathcal{O}\left(E^{\epsilon}\right) \leq 1$, moreover

a) $\mathcal{O}\left(E^{\epsilon}\right)=-2$ if and only if $v^{\epsilon}$ is not of Class $\mathbf{0}$,

b) $\mathcal{O}\left(E^{\epsilon}\right) \geq 0$ if and only if $v^{\epsilon}$ is of Class $\mathbf{0}$,

c) $\mathcal{O}\left(E^{\epsilon}\right)=1$ if and only if $v^{\epsilon}$ is of Class $\mathbf{1}$.

2. if $\mathcal{O}\left(v^{\epsilon}\right)=1$, then $0 \leq \mathcal{O}\left(E^{\epsilon}\right) \leq 2$, moreover

a) $\mathcal{O}\left(E^{\epsilon}\right) \geq 1$ if and only if $v^{\epsilon}$ is of Class $\mathbf{2}$,

b) $\mathcal{O}\left(E^{\epsilon}\right)=2$ if and only if $v^{\epsilon}$ is of Class $\mathbf{3}$, with $q=1$.

3. if $\mathcal{O}\left(v^{\epsilon}\right)=q \geq 2$, then $q-1 \leq \mathcal{O}\left(E^{\epsilon}\right) \leq q+1$, moreover

a) $\mathcal{O}\left(E^{\epsilon}\right) \geq q$ if and only if $v^{\epsilon}$ is of Class 4 ,

b) $\mathcal{O}\left(E^{\epsilon}\right)=q+1$ if and only if $v^{\epsilon}$ is of Class 3 .

REMARK 4.4. At a displacement field of a given order corresponds three possible orders of magnitude of the associated Green-Lagrange strain tensor field. Each possibility defines a class of displacements.

REMARK 4.5. The condition $\operatorname{det} F^{\epsilon}>0$ is automatically satisfied by all the displacement fields $v^{\epsilon}$ of Class 1, Class 2, Class 3 and Class 4.

We easily deduce from Theorem 4.1 useful estimates of the strain energy.

THEOREM 4.2. Let $v^{\epsilon}$ in $\mathcal{C}^{\epsilon}$ such that $\mathcal{O}\left(E^{\epsilon}\left(v^{\epsilon}\right)\right) \geq 0$. Then

$$
\mathcal{O}\left(\mathcal{W}^{\epsilon}\left(v^{\epsilon}\right)\right)=2 \mathcal{O}\left(E^{\epsilon}\left(v^{\epsilon}\right)\right) \leq 2 \mathcal{O}\left(v^{\epsilon}\right)+2 .
$$

Proof. As soon as $\mathcal{O}\left(E^{\epsilon}\left(v^{\epsilon}\right)\right)>0$, it is enough to use the development (12) of $W$ near $E=0$ to obtain the desired estimate: $\mathcal{O}\left(\mathcal{W}^{\epsilon}\left(v^{\epsilon}\right)\right)=2 \mathcal{O}\left(E^{\epsilon}\left(v^{\epsilon}\right)\right)$. If $\mathcal{O}\left(E^{\epsilon}\left(v^{\epsilon}\right)\right)=0$, then $E^{\epsilon}\left(v^{\epsilon}\right)=E^{0}+\epsilon E^{1}+\ldots$ with $E^{0} \neq 0$. Hence $\mathcal{W}^{\epsilon}\left(v^{\epsilon}\right)=$ $\int_{\Omega} W\left(E^{0}\right) \mathrm{d} x+\epsilon \mathcal{W}^{1}+\ldots$ Since $W$ vanishes only at $E=0$, we have $\int_{\Omega} W\left(E^{0}\right)$ $\mathrm{d} x>0$ and hence $\mathcal{O}\left(\mathcal{W}^{\epsilon}\left(v^{\epsilon}\right)\right)=0$. The remainder of the proof is a direct consequence of Theorem 4.1, since it was shown there that $\mathcal{O}\left(E^{\epsilon}\left(v^{\epsilon}\right)\right) \leq$ $\mathcal{O}\left(v^{\epsilon}\right)+1$.

\section{The Right Orders of Magnitude}

Let us go back to the full nonlinear problem (6): the displacement field at equilibrium $u^{\epsilon} \in \mathcal{C}^{\epsilon}$ must satisfy

$$
\mathcal{P}^{\epsilon}\left(u^{\epsilon}\right)=\min _{v \in \mathcal{C}^{\epsilon}} \mathcal{P}^{\epsilon}(v) \quad \text { with } \quad \mathcal{P}^{\epsilon}(v)=\mathcal{W}^{\epsilon}(v)-\bar{\eta} \epsilon^{n} \mathcal{L}^{\epsilon}(v)
$$


We have to find the orders of magnitude of $u^{\epsilon}, E^{\epsilon}\left(u^{\epsilon}\right), \mathcal{W}^{\epsilon}\left(u^{\epsilon}\right), \mathcal{L}^{\epsilon}\left(u^{\epsilon}\right)$ and $\mathcal{P}^{\epsilon}\left(u^{\epsilon}\right)$ for a given order of magnitude $n$ of the exterior load.

First, we construct admissible displacements whose energies have the 'right' order of magnitude. This Lemma is based on the not degenerated loading condition, i.e., Hypothesis 3.3.

LEMMA 5.1. For any $n \in \mathbb{N}$, there exists an admissible displacement $v^{\epsilon} \in \mathcal{C}^{\epsilon}$ such that

$$
\mathcal{O}\left(\epsilon^{n} \mathcal{L}^{\epsilon}\left(v^{\epsilon}\right)\right)=\mathcal{O}\left(\mathcal{P}^{\epsilon}\left(v^{\epsilon}\right)\right)=n+\max \{0 ; n-2\} .
$$

Proof. (Notation: Throughout the proof, when $v(x)=V\left(x_{3}\right), v$ will be identified with $V$.) The proof requires to consider separately the various values of $n$. In any case $V$ is chosen in $\mathcal{C}_{f l}$ in such a manner that $\ell^{0}(V) \neq 0$, which is possible thanks to hypothesis 3.3 .

Let us first consider the cases where $0 \leq n \leq 2$. We set

$$
T\left(x_{3}\right)=\left(\begin{array}{ccc}
0 & 0 & V_{1}^{\prime}\left(x_{3}\right) \\
0 & 0 & V_{2}^{\prime}\left(x_{3}\right) \\
-V_{1}^{\prime}\left(x_{3}\right) & -V_{2}^{\prime}\left(x_{3}\right) & 0
\end{array}\right)
$$

and $R_{h}\left(x_{3}\right)=e^{h T\left(x_{3}\right)}, h \in \mathbb{R}$. So, $R_{h}\left(x_{3}\right) \in S O(3)$. Let $V_{h}$ be defined by $V_{h}(0)=0$ and $V_{h}^{\prime}\left(x_{3}\right)=\left(R_{h}\left(x_{3}\right)-I\right) e_{3}$ and let $v_{h}^{\epsilon}$ be defined by

$$
v_{h}^{\epsilon}(x)=V_{h}\left(x_{3}\right)+\epsilon\left(R_{h}\left(x_{3}\right)-I\right)\left(x-x_{3} e_{3}\right) .
$$

For $h=0$, let us note that $R_{0}=I$, hence $V_{0}=0$ and $v_{0}^{\epsilon}=0$. Otherwise, when $h \neq 0$, $v_{h}^{\epsilon}$ is of Class 1. Its associated Green-Lagrange strain field is of order 1 and its leading term $E_{h}^{1}$ is given by:

$$
2 E_{h}^{1}(x)=R_{h}\left(x_{3}\right)^{T} F_{h}(x)+F_{h}(x)^{T} R_{h}\left(x_{3}\right) \quad \text { with } \quad F_{h}(x)=\left(0|0| R_{h}^{\prime}\left(x_{3}\right) x_{\alpha} e_{\alpha}\right) .
$$

Moreover, $\left.\frac{\mathrm{d} V_{h}^{\prime}}{\mathrm{d} h}\right|_{h=0}=\left.\frac{\mathrm{d}\left(R_{h}-T\right) e_{3}}{\mathrm{~d} h}\right|_{h=0}=T e_{3}=V^{\prime}$. Integrating this equality with respect to $x_{3}$ gives $\left.\frac{\mathrm{d} V_{h}}{\mathrm{~d} h}\right|_{h=0}=V$. Consequently,

$$
\left.\frac{\mathrm{d} \mathcal{L}^{0}\left(V_{h}\right)}{\mathrm{d} h}\right|_{h=0}=\ell^{0}(V) \neq 0,
$$

hence there exists $h$ such that $\mathcal{L}^{0}\left(V_{h}\right) \neq 0$.

If $n=0$ or $n=1$, thanks to Theorem 4.2, it follows that we can choose $h \neq 0$ such that $\mathcal{O}\left(\mathcal{W}^{\epsilon}\left(v_{h}^{\epsilon}\right)\right)=2>n=\mathcal{O}\left(\epsilon^{n} \mathcal{L}^{\epsilon}\left(v_{h}^{\epsilon}\right)\right)$, hence $\mathcal{O}\left(\mathcal{P}^{\epsilon}\left(v_{h}^{\epsilon}\right)\right)=\mathcal{O}\left(\epsilon^{n} \mathcal{L}^{\epsilon}\left(v_{h}^{\epsilon}\right)\right)=n$.

If $n=2$, because of Theorem 4.2, we now get $\mathcal{O}\left(\mathcal{W}^{\epsilon}\left(v_{h}^{\epsilon}\right)\right)=2 \geq$ $\mathcal{O}\left(\epsilon^{2} \mathcal{L}^{\epsilon}\left(v_{h}^{\epsilon}\right)\right)$, for any $h \neq 0$. To conclude, it should be shown that one can choose $h$ so that the two energies are of same order and not compensated. Let 
$W_{h}^{2}, L_{h}^{2}$ and $P_{h}^{2}$ be the term of order 2 of, respectively, $\mathcal{W}^{\epsilon}\left(v_{h}^{\epsilon}\right), \epsilon^{2} \mathcal{L}^{\epsilon}\left(v_{h}^{\epsilon}\right)$ and $\mathcal{P}^{\epsilon}\left(v_{h}^{\epsilon}\right)$. They are given by

$$
W_{h}^{2}=\frac{1}{2} \int_{\Omega} C E_{h}^{1} \cdot E_{h}^{1} \mathrm{~d} x, \quad L_{h}^{2}=\mathcal{L}^{0}\left(V_{h}\right), \quad P_{h}^{2}=W_{h}^{2}-\bar{\eta} L_{h}^{2} .
$$

Since $F_{0}=E_{0}{ }^{1}=0$, we obtain

$$
\left.\frac{\mathrm{d} W_{h}^{2}}{\mathrm{~d} h}\right|_{h=0}=0,\left.\quad \frac{\mathrm{d} L_{h}^{2}}{\mathrm{~d} h}\right|_{h=0}=\ell^{0}(V) \neq 0,\left.\quad \frac{\mathrm{d} P_{h}^{2}}{\mathrm{~d} h}\right|_{h=0}=-\bar{\eta} \ell^{0}(V) \neq 0
$$

which is sufficient to conclude that we can choose $h$ such that $\mathcal{O}\left(\mathcal{P}^{\epsilon}\left(v_{h}^{\epsilon}\right)\right)=$ $\mathcal{O}\left(\epsilon^{2} \mathcal{L}^{\epsilon}\left(v_{h}^{\epsilon}\right)\right)=2$.

Let us now consider the case where $n \geq 3$. Let us set, for $h \in \mathbb{R}$,

$$
v_{h}^{\epsilon}(x)=h \epsilon^{n-2} V_{\alpha}\left(x_{3}\right) e_{\alpha}-h \epsilon^{n-1} x_{\alpha} V_{\alpha}^{\prime}\left(x_{3}\right) e_{3} .
$$

So, $v_{0}^{\epsilon}=0, v_{h}^{\epsilon}$ is of order $n-2$ and of Class 3 for any $h \neq 0$. Developing $\mathcal{L}^{\epsilon}\left(v^{\epsilon}\right)$ leads to

$$
\mathcal{L}^{\epsilon}\left(v_{h}^{\epsilon}\right)=\mathcal{L}^{0}\left(v_{h}^{\epsilon}\right)+\epsilon \mathcal{L}^{1}\left(v_{h}^{\epsilon}\right)+\ldots=h \epsilon^{n-2} \ell^{0}(V)+o\left(\epsilon^{n-2}\right)
$$

which implies that, for any $\left.h \neq 0, \epsilon^{n} \mathcal{L}^{\epsilon}\left(v_{h}^{\epsilon}\right)\right)$ is of order $2 n-2$ and its leading term is $L_{h}^{2 n-2}=h \ell^{0}(V)$. By virtue of Theorem 4.1, $E^{\epsilon}\left(v_{h}^{\epsilon}\right)$ is of order $n-1$ and its leading term $E_{h}^{n-1}$ is given by:

$$
\text { When } n=3: \quad E_{h}^{2}=-\frac{h^{2}}{2} V^{\prime} \otimes V^{\prime}-\left(h V^{\prime \prime} \cdot x+\frac{h^{2}}{2} V^{\prime} \cdot V^{\prime}\right) e_{3} \otimes e_{3} \text {, }
$$

When $n>3: E_{h}^{n-1}=-h V^{\prime \prime} \cdot x e_{3} \otimes e_{3}$.

Consequently $\mathcal{W}^{\epsilon}\left(v_{h}^{\epsilon}\right)$ is of order $2 n-2$ and its leading term is $W_{h}^{2 n-2}=$ $\frac{1}{2} \int_{\Omega} C E_{h}^{n-1} \cdot E_{h}^{n-1} \mathrm{~d} x$. Since $E_{0}^{n-1}=0$, we obtain

$$
\left.\frac{\mathrm{d} W_{h}^{2 n-2}}{\mathrm{~d} h}\right|_{h=0}=0,\left.\quad \frac{\mathrm{d} L_{h}^{2 n-2}}{\mathrm{~d} h}\right|_{h=0}=\ell^{0}(V) \neq 0,\left.\quad \frac{\mathrm{d} P_{h}^{2 n-2}}{\mathrm{~d} h}\right|_{h=0}=-\bar{\eta} \ell^{0}(V) \neq 0
$$

which is sufficient to conclude that we can choose $h$ such that $\mathcal{O}\left(\mathcal{P}^{\epsilon}\left(v_{h}^{\epsilon}\right)\right)=$ $\mathcal{O}\left(\epsilon^{n} \mathcal{L}^{\epsilon}\left(v_{h}^{\epsilon}\right)\right)=2 n-2$.

The following theorem is the main result of this section. It gives the order of magnitude of the displacement field and the energies associated with a stable equilibrated configuration, according to the order of magnitude of the loading. 
THEOREM 5.2. The right orders of magnitude are given in the following table

\begin{tabular}{llllllll}
\hline Case & $n$ & $\mathcal{O}\left(u^{\epsilon}\right)$ & $u^{\epsilon}$ & $\mathcal{O}\left(E^{\epsilon}\left(u^{\epsilon}\right)\right)$ & $\mathcal{O}\left(\mathcal{W}^{\epsilon}\left(u^{\epsilon}\right)\right)$ & $\mathcal{O}\left(\epsilon^{n} \mathcal{L}^{\epsilon}\left(u^{\epsilon}\right)\right)$ & $\mathcal{O}\left(\mathcal{P}^{\epsilon}\left(u^{\epsilon}\right)\right)$ \\
\hline (i) & 0 & 0 & Class 0 & $\geq 0$ & $\geq 0$ & 0 & 0 \\
(ii) & 1 & 0 & Class 1 & 1 & 2 & 1 & 1 \\
(iii) & 2 & 0 & Class 1 & 1 & 2 & 2 & 2 \\
(iv) & $\geq 3$ & $n-2$ & Class 3 & $n-1$ & $2 n-2$ & $2 n-2$ & $2 n-2$ \\
\hline
\end{tabular}

Proof. By virtue of equations (16) and (26), we already know that $\mathcal{O}\left(u^{\epsilon}\right) \geq 0$ and that

$$
\begin{aligned}
n+\max \{0 ; n-2\} & \geq \mathcal{O}\left(\mathcal{P}^{\epsilon}\left(u^{\epsilon}\right)\right) \geq \mathcal{O}\left(\epsilon^{n} \mathcal{L}^{\epsilon}\left(u^{\epsilon}\right)\right) \\
2 \mathcal{O}\left(u^{\epsilon}\right)+2 \geq 2 \mathcal{O}\left(E^{\epsilon}\left(u^{\epsilon}\right)\right) & =\mathcal{O}\left(\mathcal{W}^{\epsilon}\left(u^{\epsilon}\right)\right) \geq \mathcal{O}\left(\epsilon^{n} \mathcal{L}^{\epsilon}\left(u^{\epsilon}\right)\right) \\
& \geq n+\mathcal{O}\left(u^{\epsilon}\right)
\end{aligned}
$$

Case $n=0$. Then equations (32) and (33) give $\mathcal{O}\left(u^{\epsilon}\right)=\mathcal{O}\left(\mathcal{P}^{\epsilon}\left(u^{\epsilon}\right)\right)=$ $\mathcal{O}\left(\epsilon^{0} \mathcal{L}^{\epsilon}\left(u^{\epsilon}\right)\right)=0,1 \geq \mathcal{O}\left(E^{\epsilon}\left(u^{\epsilon}\right)\right) \geq 0$ and $2 \geq \mathcal{O}\left(\mathcal{W}^{\epsilon}\left(u^{\epsilon}\right)\right) \geq 0$. Hence Theorem 4.1 says that $u^{\epsilon}$ is of Class $\mathbf{0}$, what proves (i).

Case $n=1$. Then equations (32) and (33) give $\mathcal{O}\left(\mathcal{P}^{\epsilon}\left(u^{\epsilon}\right)\right)=\mathcal{O}\left(\epsilon^{1} \mathcal{L}^{\epsilon}\left(u^{\epsilon}\right)\right)=$ $1, \mathcal{O}\left(u^{\epsilon}\right)=0$ and $2 \geq 2 \mathcal{O}\left(E^{\epsilon}\left(u^{\epsilon}\right)\right)=\mathcal{O}\left(\mathcal{W}^{\epsilon}\left(u^{\epsilon}\right)\right) \geq 1$. The unique possibility is that $\mathcal{O}\left(E^{\epsilon}\left(u^{\epsilon}\right)\right)=1$. Hence $\mathcal{O}\left(\mathcal{W}^{\epsilon}\left(u^{\epsilon}\right)\right)=2$ and, by virtue of Theorems $4.1, u^{\epsilon}$ is of Class 1, thus (ii).

Case $n=2$. Then equations (32) and (33) give $\mathcal{O}\left(\mathcal{P}^{\epsilon}\left(u^{\epsilon}\right)\right)=\mathcal{O}\left(\epsilon^{2} \mathcal{L}^{\epsilon}\left(u^{\epsilon}\right)\right)=$ 2, $\mathcal{O}\left(u^{\epsilon}\right)=0, \mathcal{O}\left(E^{\epsilon}\left(u^{\epsilon}\right)\right)=1$ and $\mathcal{O}\left(\mathcal{W}^{\epsilon}\left(u^{\epsilon}\right)\right)=2$. By virtue of Theorems 4.1, $u^{\epsilon}$ is of Class $\mathbf{1}$, hence (iii).

Case $n \geq 3$. Then equations (32) and (33) give $\mathcal{O}\left(\mathcal{P}^{\epsilon}\left(u^{\epsilon}\right)\right)=\mathcal{O}\left(\epsilon^{n} \mathcal{L}^{\epsilon}\left(u^{\epsilon}\right)\right)=$ $\mathcal{O}\left(\mathcal{W}^{\epsilon}\left(u^{\epsilon}\right)\right)=2 n-2, \mathcal{O}\left(u^{\epsilon}\right)=n-2$ and $\mathcal{O}\left(E^{\epsilon}\left(u^{\epsilon}\right)\right)=n-1$. By virtue of Theorems 4.1, $u^{\epsilon}$ is of Class 3, what proves (iv).

REMARK 5.1. We note that the displacements are of order 0 as soon as the order of the load is less than or equal to 2 . Moreover we see that the order of the elastic energy is always equal to the order of the potential energy except when $n=1$, for which it is negligible.

We also note that $U_{3}$ is equal to zero as soon as $n \geq 3$. Moreover $U$ corresponds to an inextensional displacement when $n=1$ or $n=2$.

\section{Hierarchy of One-dimensional Models}

In order to find the limit models associated to the different orders of the exterior load, we proceed as follows:

1. We fix $n$ and choose admissible displacement fields $v^{\epsilon}$ of the same class and of the same order as those obtained for $u^{\epsilon}$ in Theorem 5.2. 
2. We express the leading term of the potential energy $\mathcal{P}^{\epsilon}\left(v^{\epsilon}\right)$, say $\mathcal{P}^{m}$ with $m=$ $n+\max \{0, n-2\}$, in terms of the involved two or three first terms of the expansion of $v^{\epsilon}$, say $v^{q}(x)=V\left(x_{3}\right), v^{q+1}$ and eventually $v^{q+2}$ with $q=\max \{0$, $n-2\}$.

3. We eventually minimize $\mathcal{P}^{m}$ with respect to $v^{q+2}$, at given $V$ and $v^{q+1}$; then, we minimize $\mathcal{P}^{m}$ - which is then considered as a functional of $V$ and $v^{q+1}$ with respect to $v^{q+1}$ at given $V$.

4. We then obtain $\mathcal{P}^{m}$ as a functional of the leading term $V$ alone. Its minimization constitutes the desired one-dimensional model.

Throughout this section, we denote by $|\omega|$ and $I_{\alpha \beta}$, respectively, the area and the geometrical moments of the dimensionless cross-section $\omega$, and we assume that the origin of the coordinates corresponds to the centroid of $\omega$

$$
|\omega|=\int_{\omega} \mathrm{d} x_{1} \mathrm{~d} x_{2}, \quad \int_{\omega} x_{\alpha} \mathrm{d} x_{1} \mathrm{~d} x_{2}=0, \quad I_{\alpha \beta}=\int_{\omega} x_{\alpha} x_{\beta} \mathrm{d} x_{1} \mathrm{~d} x_{2} .
$$

We start by considering the weakest loadings and we finish by the loadings of order 0 . Thus the reader will be able to see the evolution of the behavior of the body by imagining that the loading is increased gradually.

\subsection{CASE $n \geq 4$}

Step 1. Here $m=2 n-2$ and $q=n-2$. Since $u^{\epsilon}$ is of Class 3 and of order $n-2$, its first two leading terms read as

$$
\begin{aligned}
& u^{n-2}(x)=U_{\alpha}\left(x_{3}\right) e_{\alpha}, \\
& u^{n-1}(x)=\bar{\theta}_{u}\left(x_{3}\right) e_{3 \wedge} x-x_{\alpha} U_{\alpha}^{\prime}\left(x_{3}\right) e_{3}+\bar{U}\left(x_{3}\right) .
\end{aligned}
$$

We choose $v^{\epsilon}$ with the same form, i.e.

$$
u^{n-2}(x)=V_{\alpha}\left(x_{3}\right) e_{\alpha}, \quad v^{n-1}(x)=\bar{\theta}_{v}\left(x_{3}\right) e_{3 \wedge} x-x_{\alpha} V_{\alpha}^{\prime}\left(x_{3}\right) e_{3}+\bar{V}\left(x_{3}\right) .
$$

The leading terms $V$ and $U$ belong to $\mathcal{C}_{f l}$.

Step 2. The leading term of the Green-Lagrange strain field, say $E^{n-1}$, is given by

$$
\begin{aligned}
2 E_{\alpha \beta}^{n-1} & =v_{\beta, \alpha}^{n}+v_{\alpha, \beta}^{n}, \quad 2 E_{\alpha 3}^{n-1}=\bar{V}_{\alpha}^{\prime}+\bar{\theta}_{v}^{\prime}\left(e_{3 \wedge} x\right) \cdot e_{\alpha}+v_{3, \alpha}^{n}, \\
E_{33}^{n-1} & =\bar{V}_{3}^{\prime}-x_{\alpha} V_{\alpha}^{\prime \prime} .
\end{aligned}
$$

The leading term of the Piola-Kirchhoff stress field is $\Sigma^{n-1}=C E^{n-1}$. As $E^{n-1}$ depends on the first three terms of the expansion of $v^{\epsilon}$, it is also the case for the leading term of the potential energy

$$
\mathcal{P}^{2 n-2}=\frac{1}{2} \int_{\Omega} C E^{n-1} \cdot E^{n-1} \mathrm{~d} x-\bar{\eta} \ell^{0}(V) .
$$


This dependence is quadratic. Since the remainder of the procedure is classic, the broad outline only is given, see [22] for details.

Step 3. Minimizing $\mathcal{P}^{2 n-2}$ with respect to $v^{n}$ leads to the famous Saint-Venant problems of extension, bending and torsion. Since the cross-section is homogeneous and since the material is isotropic, these problems are uncoupled. Moreover the transversal components $\sum_{\alpha \beta}^{n-1}$ of the Piola-Kirchhoff stress tensor vanish. The solution of the extension and bending problems can be obtained in a closed form, while that is possible for the torsion problem only for very particular forms of section. Finally, we obtain $v^{n}$ up to 'rigid displacements':

$$
\begin{aligned}
& v_{1}^{n}(x)=V_{1}^{\prime \prime}\left(x_{3}\right) \frac{\nu}{2}\left(x_{1}^{2}-x_{2}^{2}\right)+V_{2}^{\prime \prime}\left(x_{3}\right) \nu x_{1} x_{2}-\bar{V}_{3}^{\prime}\left(x_{3}\right) \nu x_{1}, \\
& v_{2}^{n}(x)=V_{1}^{\prime \prime}\left(x_{3}\right) \nu x_{1} x_{2}+V_{2}^{\prime \prime}\left(x_{3}\right) \frac{\nu}{2}\left(x_{2}^{2}-x_{1}^{2}\right)-\bar{V}_{3}^{\prime}\left(x_{3}\right) \nu x_{2}, \\
& v_{3}^{n}(x)=-\bar{V}_{\alpha}^{\prime}\left(x_{3}\right) x_{\alpha}+\bar{\theta}_{v}^{\prime}\left(x_{3}\right) w\left(x_{1}, x_{2}\right),
\end{aligned}
$$

where $w$ denotes the solution of the torsion problem,

$$
\int_{\omega}\left(\left(w_{, 1}-x_{2}\right) \phi_{, 1}+\left(w_{, 2}+x_{1}\right) \phi_{, 2}\right) \mathrm{d} x_{1} \mathrm{~d} x_{2}=0, \quad \forall \phi \in H^{1}(\omega) .
$$

After inserting these results into $\mathcal{P}^{2 n-2}$, the leading term of the potential energy becomes

$$
\mathcal{P}^{2 n-2}=\frac{1}{2} \int_{2}^{1}\left(|\omega| \bar{V}_{3}^{\prime 2}+J \bar{\theta}_{v}^{\prime 2}+I_{\alpha \beta} V_{\alpha}^{\prime \prime} V_{\beta}^{\prime \prime}\right) \mathrm{d} x_{3}-\bar{\eta} \ell^{0}(V)
$$

where $J$ denotes the torsion rigidity modulus of the cross-section,

$$
J=\int_{\omega}\left(x_{1}^{2}+x_{2}^{2}-\nabla w \cdot \nabla w\right) \mathrm{d} x_{1} \mathrm{~d} x_{2} .
$$

In equation (40), the strain energy is the sum of three terms: the first corresponds to the extension energy, the second to the torsion energy and the third to the bending energy. But, since the displacements fields $\bar{V}_{3}$ and $\bar{\theta}_{v}$ are not involved in the leading term of the loading potential $\ell^{0}$, the minimization of $\mathcal{P}^{2 n-2}$ with respect to $\bar{V}_{3}$ and $\bar{\theta}_{v}$, at given $U$, gives

$$
\bar{U}_{3}=0, \quad \bar{\theta}_{u}=0 .
$$

Consequently, the extension and torsion energies are negligible in comparison to the bending energy. 
Step 4. Finally, the leading term of the displacement field $U$ and of the potential energy are given by solving the following minimization problem:

Find $U \in \mathcal{C}_{f l}$ minimizing $\frac{1}{2} \int_{0}^{1} I_{\alpha \beta} V_{\alpha}^{\prime \prime}\left(x_{3}\right) V_{\beta}^{\prime \prime}\left(x_{3}\right) \mathrm{d} x_{3}-\bar{\eta} \ell^{0}(V)$ on $\mathcal{C}_{f l}$.

REMARK 6.1. Here one recognizes the usual model of elastic bending of the linear theory of beams. It is the reign of linearity: the displacements and the strains are small, the forces act only like dead loads, and the theorems of uniqueness prevail.

\section{2. $\operatorname{CASE} n=3$}

Step 1. Here $m=4$ and $q=1$. Since $u^{\epsilon}$ is of Class 3 and of order 1, its first two leading terms take the form

$$
u^{1}(x)=U_{\alpha}\left(x_{3}\right) e_{\alpha}, \quad u^{2}(x)=\bar{\theta}_{u}\left(x_{3}\right) e_{3 \wedge} x-x_{\alpha} U_{\alpha}^{\prime}\left(x_{3}\right) e_{3}+\bar{U}\left(x_{3}\right) .
$$

We choose $v^{\epsilon}$ with the same form, i.e.

$$
v^{1}(x)=V_{\alpha}\left(x_{3}\right) e_{\alpha}, \quad v^{2}(x)=\bar{\theta}_{v}\left(x_{3}\right) e_{3 \wedge} x-x_{\alpha} V_{\alpha}^{\prime}\left(x_{3}\right) e_{3}+\bar{V}\left(x_{3}\right) .
$$

The leading terms $V$ and $U$ belong still to $\mathcal{C}_{f l}$.

Step 2. The leading term $E^{2}$ of the Green-Lagrange strain field is given by

$$
\begin{aligned}
2 E_{\alpha \beta}^{2} & =v_{\beta, \alpha}^{3}+v_{\alpha, \beta}^{3}+V_{\alpha}^{\prime} V_{\beta}^{\prime}, \quad 2 E_{\alpha 3}^{2}=\bar{V}_{\alpha}^{\prime}+\bar{\theta}_{v}^{\prime}\left(e_{3 \wedge} x\right) \cdot e_{\alpha}+v_{3, \alpha}^{3}, \\
E_{33}^{2} & =\bar{V}_{3}^{\prime}+\frac{1}{2} V_{\alpha}^{\prime} V_{\alpha}^{\prime}-x_{\alpha} V_{\alpha}^{\prime \prime},
\end{aligned}
$$

the leading term of the Piola-Kirchhoff stresses is $\Sigma^{2}=C E^{2}$ and the leading term of the potential energy reads as

$$
\mathcal{P}^{4}=\frac{1}{2} \int_{\Omega} C E^{2} \cdot E^{2} \mathrm{~d} x-\bar{\eta} \ell^{0}(V) .
$$

This functional is no more quadratic, because of the appearance of nonlinear terms such $V_{\alpha}^{\prime} V_{\beta}^{\prime}$. However the results differ little from those of the preceding case.

Step 3. Minimizing $\mathcal{P}^{4}$ with respect to $v^{n}$ leads to the Saint-Venant problems again. The transversal components $\Sigma_{\alpha \beta}^{2}$ of the leading term of the Piola- 
Kirchhoff stress tensor still vanish. The torsion problem remains unchanged. Finally, the leading term of the potential energy reads

$$
\mathcal{P}^{4}=\frac{1}{2} \int_{0}^{1}\left(|\omega|\left(\bar{V}_{3}^{\prime}+\frac{1}{2} V_{\alpha}^{\prime} V_{\alpha}^{\prime}\right)^{2}+J \bar{\theta}_{v}^{\prime 2}+I_{\alpha \beta} V_{\alpha}^{\prime \prime} V_{\beta}^{\prime \prime}\right) \mathrm{d} x_{3}-\bar{\eta} \ell^{0}(V) .
$$

Thus the extension energy only is different. But, since the displacements fields $\overline{V_{3}}$ and $\bar{\theta}_{v}$ are still not involved in the leading term of the loading potential, the minimization of $\mathcal{P}^{2 n-2}$ with respect to $\bar{V}_{3}$ and $\bar{\theta}_{v}$, at given $U$, gives

$$
\bar{U}_{3}^{\prime}=-\frac{1}{2} U_{\alpha}^{\prime} U_{\alpha}^{\prime}, \quad \bar{\theta}_{u}=0
$$

and hence the extension and torsion energies are still negligible in comparison to the bending energy.

Step 4. Finally, the leading term of the displacement field $U$ is given by solving still Equation (43).

REMARK 6.2. This model corresponds to the model obtained by $\Gamma$-convergence by [26].

\subsection{CASE $n=2$}

Step 1. Here $m=2$ and $q=0$, the displacements are finite, but the strains are still infinitesimal. Since $u^{\epsilon}$ is of Class 1 and of order 0, its first two leading terms take the form

$$
\begin{aligned}
& u^{0}(x)=U\left(x_{3}\right), \quad u^{1}(x)=x_{\alpha}\left(R\left(x_{3}\right)-I\right) e_{\alpha}+\bar{U}\left(x_{3}\right), \\
& R\left(x_{3}\right) \in S O(3), R(0)=I, \quad U^{\prime}=(R-I) e_{3}, U(0)=0 .
\end{aligned}
$$

We choose $v^{\epsilon}$ with the same form, i.e.,

$$
\begin{aligned}
& v^{0}(x)=V\left(x_{3}\right), \quad v^{1}(x)=x_{\alpha}\left(Q\left(x_{3}\right)-I\right) e_{\alpha}+\bar{V}\left(x_{3}\right), \\
& Q\left(x_{3}\right) \in S O(3), Q(0)=I, \quad V^{\prime}=(Q-I) e_{3}, V(0)=0 .
\end{aligned}
$$

Step 2. The leading term $E^{1}$ of the Green-Lagrange strain field is given by

$$
\begin{aligned}
& 2 E_{\alpha \beta}^{1}=\left(Q^{T} v^{2}\right)_{\beta, \alpha}+\left(Q^{T} v^{2}\right)_{\alpha, \beta}, \\
& 2 E_{\alpha 3}^{1}=\left(Q^{T} v^{2}\right)_{3, \alpha}+x_{\beta} Q_{i \alpha} Q_{i \beta}^{\prime}+Q_{i \alpha} \bar{V}_{i}^{\prime},
\end{aligned}
$$




$$
E_{33}^{1}=x_{\beta} Q_{i 3} Q_{i \beta}^{\prime}+Q_{i 3} \bar{V}_{i}^{\prime} .
$$

The leading term of the Piola-Kirchhoff stresses is $\Sigma^{1}=C E^{1}$ and the leading term of the potential energy reads as

$$
\mathcal{P}^{2}=\frac{1}{2} \int_{\Omega} C E^{1} \cdot E^{1} \mathrm{~d} x-\bar{\eta} \mathcal{L}^{0}(V) .
$$

This functional is quadratic in terms of $v^{2}$ and $\bar{V}$, for a given $Q$.

Step 3. Minimizing $\mathcal{P}^{2}$ with respect to $v^{2}$ involves the Saint-Venant problems once more. The transversal components $\Sigma_{\alpha \beta}^{1}$ of the leading term of the PiolaKirchhoff stress tensor still vanish. Because of the linearity, we find

$$
\begin{aligned}
\left(Q^{T} v^{2}\right)_{1}(x)= & \left(Q^{T} Q^{\prime}\right)_{13}\left(x_{3}\right) \frac{\nu}{2}\left(x_{1}^{2}-x_{2}^{2}\right)+\left(Q^{T} Q^{\prime}\right)_{23}\left(x_{3}\right) \nu x_{1} x_{2} \\
& -\left(Q^{T} \bar{V}^{\prime}\right)_{3}\left(x_{3}\right) \nu x_{1}, \\
\left(Q^{T} v^{2}\right)_{2}(x)= & \left(Q^{T} Q^{\prime}\right)_{13}\left(x_{3}\right) \nu x_{1} x_{2}+\left(Q^{T} Q^{\prime}\right)_{23}\left(x_{3}\right) \frac{\nu}{2}\left(x_{2}^{2}-x_{1}^{2}\right) \\
& -\left(Q^{T} \bar{V}^{\prime}\right)_{3}\left(x_{3}\right) \nu x_{2}, \\
\left(Q^{T} v^{2}\right)_{3}(x)= & -\left(Q^{T} \bar{V}^{\prime}\right)_{\alpha}\left(x_{3}\right) x_{\alpha}+\left(Q^{T} Q^{\prime}\right)_{21}\left(x_{3}\right) w\left(x_{1}, x_{2}\right) .
\end{aligned}
$$

After insertion into the potential energy equation, we get

$$
\mathcal{P}^{2}=\frac{1}{2} \int_{0}^{1}\left(|\omega|\left(Q^{T} \bar{V}^{\prime}\right)_{3}^{2}+J\left(Q^{T} Q^{\prime}\right)_{12}^{2}+I_{\alpha \beta}\left(Q^{T} Q^{\prime}\right)_{3 \alpha}\left(Q^{T} Q^{\prime}\right)_{3 \beta}\right) \mathrm{d} x_{3}-\bar{\eta} \mathcal{L}^{0}(V) .
$$

At $Q$ fixed, the minimization of $\mathcal{P}^{2}$ with respect to $\bar{V}$ give (for $Q=R$ ):

$$
\left(R^{T} \bar{U}\right)_{3}=0
$$

and hence the extension energy is once more negligible.

Step 4. To determine the leading term $U$ of the displacement, in practise, it is more convenient to consider the skew-symmetric matrix field $S=R^{T} R^{\prime}$ as the principal unknown. Indeed, let

$$
\mathcal{S}=\left\{T \in H^{1}((0,1) ; S k(3)) \mid T(0)=0\right\} .
$$

Then, a unique field $Q_{T}$ such that $Q_{T}(0)=I$ and $Q_{T}^{T} Q_{T}^{\prime}=T$ is associated with each $T \in \mathcal{S}$. Thus $Q_{T}$ is an admissible rotation field. Moreover, let $\mathcal{V}$ be the functional 
which associates to $T$ the displacement field $V=\mathcal{V}(T)$ defined by $V^{\prime}=\left(Q_{T}-\right.$ I) $e_{3}$ and $V(0)=0$. Then, the leading term of the potential energy can be considered as the following functional of $T$ :

$$
\mathcal{P}^{2}(T)=\frac{1}{2} \int_{0}^{1}\left(J T^{\prime 2}{ }_{12}^{2}+I_{\alpha \beta} T^{\prime}{ }_{3 \alpha} T^{\prime}{ }_{3 \beta}\right) d x_{3}-\bar{\eta} \mathcal{L}^{0} \circ \mathcal{V}(T) .
$$

Its minimization constitutes the desired one-dimensional model

$$
\text { Find } S \in \mathcal{S} \text { minimizing } \mathcal{P}^{2}(T) \text { for } T \in \mathcal{S} \text {. }
$$

Once the minimizer $S$ is found, we obtain $U=\mathcal{V}(S)$. This the non-linear model of inextensible, elastically flexible beams.

REMARK 6.3. The leading term of the strain energy is quadratic because the strains are small, but the potential of the external forces remains or becomes nonlinear. Thus, it is the first genuinely nonlinear model. It is the model which contains the phenomena of buckling. It is the only model where the strain energy is the sum of a bending energy and of a torsion energy. This model corresponds to the model obtained by $\Gamma$-convergence by [25].

\subsection{CASE $n=1$}

Here $m=1$ and $q=0$, the displacements are finite, but the strains are still infinitesimal. Moreover, the strain energy is negligible in comparison to the loading potential. The displacement solution $u^{\epsilon}$ is of Class 1: $u^{0}(x)=U\left(x_{3}\right)$ with $U^{\prime}=(R-I) e_{3}, U(0)=0$ and $R\left(x_{3}\right) \in S O(3)$. If we choose $v^{\epsilon}$ of the same form, i.e., $v^{0}(x)=V\left(x_{3}\right)$ with $V^{\prime}=(Q-I) e_{3}, V(0)=0$ and $Q\left(x_{3}\right) \in S O(3)$, the leading term of the potential energy $\mathcal{P}^{1}$ is reduced to $-\bar{\eta} \mathcal{L}^{0} V$. It involves $V$ only which, because of its link with the rotation field $Q$, must satisfy the inextensional constraint: $\left\|e_{3}+V^{\prime}\right\|=1$. Finally, the one-dimensional problem reads

$$
\text { Find } U \text { minimizing }-\bar{\eta} \mathcal{L}^{0}(V) \quad \text { on } \quad\left\{V:\left\|e_{3}+V^{\prime}\right\|=1, V(0)=0\right\} .
$$

This is the model of inextensional strings.

REMARK 6.4. It is the only model where the strain energy is negligible in comparison to the potential energy due to the external forces. The body has lost the memory of the rectilinear character of its natural configuration and remembers only its natural length. At this level of loading, all the phenomena of buckling already took place. 


\subsection{CASE $n=0$}

Steps 1-2. In this case $m=q=0$. Choosing $v^{\epsilon}$ of Class $\mathbf{0}$ and of order 0 , we get $v^{0}(x)=V\left(x_{3}\right)$ with $V(0)=0$. The leading term of the Green-Lagrange strain field depends only on $V$ and $v^{1}$ :

$$
2 E^{0}=F^{0^{T}} F^{0}-I, \quad F^{0}=\left(e_{1}+v_{, 1}^{1}\left|e_{2}+v_{, 2}^{1}\right| e_{3}+V^{\prime}\right),
$$

and $\left(V, v^{1}\right)$ must satisfy the condition $\operatorname{det} F^{0}>0$. It is more convenient for the sequel to consider the strain energy density as a function of $F^{0}$ :

$$
W\left(E^{0}\right) \equiv \bar{W}\left(F^{0}\right) .
$$

The leading term of the potential energy can read as:

$$
\mathcal{P}^{0}\left(v^{1}, V\right)=\int_{\Omega} \bar{W}\left(e_{1}+v_{, 1}^{1}(x), e_{2}+v_{, 2}^{1}(x), e_{3}+V^{\prime}\left(x_{3}\right)\right) \mathrm{d} x-\bar{\eta} \mathcal{L}^{0}(V) .
$$

Step 3. Let us first minimize $\mathcal{P}^{0}$ with respect to $v^{1}$ at given $V$. That is equivalent to minimize the strain energy with respect to $v^{1}$ at given $V$. But, since $v^{1}$ is only involved through its derivatives with respect to $\left(x_{1}, x_{2}\right)$, the coordinate $x_{3}$ plays the role of a parameter and we can localize the minimization on each crosssection. Thus the generic problem becomes:

$$
\inf _{\left\{v: \omega \rightarrow \mathbb{R}^{3} \mid \operatorname{det}\left(e_{1}+v_{1}\left|e_{2}+v_{2}\right| c\right)>0\right\}} \int_{\omega} \bar{W}\left(e_{1}+v_{, 1}, e_{2}+v_{, 2}, c\right) \mathrm{d} x_{1} \mathrm{~d} x_{2}
$$

for a given $c \in \mathbb{R}^{3}$. But, since the cross-section is homogeneous, this problem can be localized again and becomes a problem of minimization on $\mathbb{R}^{6}$ :

$$
W^{1}(c)=\inf _{(a, b) \in \mathbb{R}^{6}: \operatorname{det}(a|b| c)>0} \bar{W}(a, b, c) .
$$

Thus, we have obtained

$$
\inf _{v^{1}} \mathcal{P}^{0}\left(v^{1}, V\right)=\int_{0}^{1}|\omega| W^{1}\left(e_{3}+V^{\prime}\left(x_{3}\right)\right) \mathrm{d} x_{3}-\bar{\eta} \mathcal{L}^{0}(V) .
$$

The one-dimensional elastic potential $W^{1}$ enjoys the following properties: (i) Since $\bar{W}$ is an isotropic function, so is $W^{1}$ which thus depends on $c$ only through $\|c\|$; (ii) Since $\bar{W}(F)$ is non-negative and vanishes if and only if $F$ is a rotation, $W^{1}$ is non-negative and vanishes if and only if $\|c\|=1$; (iii) Consequently, $W^{1}$ is not convex; (iv) Its convex envelope, say $c \mapsto W^{* *}(c)$, vanishes if and only if $\|c\| \leq 1$, i.e., when the string is in compression; (v) In the case of the Saint- 
Venant Kirchhoff potential, i.e., when $W(E)=\frac{1}{2} C E \cdot E$, one obtains an explicit expression of $W^{1}$, see [23]:

$$
W^{1}(c)=\frac{1-\nu}{8(1+\nu)(1-2 \nu)}\left(\|c\|^{2}-\frac{1+\nu}{1-\nu}\right)^{2}+\frac{1}{4(1-\nu)}-\frac{\left\langle 1+\nu-\nu\|c\|^{2}\right\rangle^{2}}{4(1+\nu)(1-2 \nu)},
$$

where $\langle\cdot\rangle$ denotes the positive part.

Step 4. Finally the problem reads as

$$
\text { Find } U \text { minimizing } \int_{0}^{1}|\omega| W^{1}\left(e_{3}+V^{\prime}\left(x_{3}\right)\right) \mathrm{d} x_{3}-\bar{\eta} \mathcal{L}^{0}(V) \text { on }\{V: V(0)=0\} .
$$

This is the model of nonlinear elastic strings.

REMARK 6.5. It is the only model in which the nonlinear behavior of the material plays a role. The strain energy is, at most, of the order of the potential energy of the external forces. But, according to the type of loading, it could happen that the body behaves like an inextensible string. In other words, it can happen that the solution of Equation (65) satisfies $\left\|e_{3}+U^{\prime}\right\| \leq 1$. In such a case, the extension energy is negligible. This explains why the order of the strains is not perfectly given in Theorem 4.1 when $n=0$.

REMARK 6.6. Since $W^{1}$ is not convex, the problem (65) must be relaxed. In the present context of a one-dimensional problem, that simply consists in changing $W^{1}$ by its convex envelope $W^{* *}$, see [10]. The relaxed model was first deduced, by $\Gamma$-convergence, from the three-dimensional theory of elasticity by [1].

\section{Appendix}

PROOF OF THEOREM 4.1

For clarity, we divide the proof of theorem 4.1 into several lemmas.

LEMMA A.1. Let $x \mapsto v(x)$ and $x_{3} \mapsto V\left(x_{3}\right)$ be smooth vector fields such that

$$
R(x)=\left(e_{1}+v_{, 1}(x)\left|e_{2}+v_{, 2}\right| e_{3}+V^{\prime}\left(x_{3}\right)\right) \in S O(3), \quad \forall x \in \Omega .
$$

Then $R(x)$ depends only on $x_{3}$ and the fields $v$ and $V$ satisfy

$$
V^{\prime}\left(x_{3}\right)=\left(R\left(x_{3}\right)-I\right) e_{3}, \quad v(x)=\left(R\left(x_{3}\right)-I\right)\left(x_{\alpha} e_{\alpha}\right)+\bar{V}\left(x_{3}\right) .
$$


Proof. Let us prove that $R_{, 1}=0$. Since $R e_{3}=e_{3}+V^{\prime}$, we have $R_{, 1} e_{3}=0$. Since $R e_{1} \cdot R e_{1}=1$ and $R e_{1} \cdot R e_{3}=0$, we get $R_{, 1} e_{1} \cdot R e_{1}=R_{, 1} e_{1} \cdot R e_{3}=0$. Since $R e_{1} \cdot R e_{2}=0$, we get $R_{, 1} e_{1} \cdot R e_{2}+R_{, 1} e_{2} \cdot R e_{1}=0$. But, since $v_{, 12}=v_{, 21}$, we have also $R_{, 1} e_{2}=R_{, 2} e_{1}$. Hence $R_{, 1} e_{1} \cdot R_{2}=0$. But then, since $R_{, 1} e_{1}$ is orthogonal to all the $R e_{i}{ }^{\prime} \mathrm{s}, R_{, 1} e_{1}=0$. Consequently, $R_{, 1} e_{2} \cdot R e_{1}=0$. But, since $R e_{2} \cdot R e_{2}=1$ and $R e_{2} \cdot R e_{3}=0$, we have also $R_{, 1} e_{2} \cdot R e_{2}=R_{, 1} e_{2} \cdot R e_{3}=0$. Thus $R_{, 1} e_{2}=0$ and finally $R_{, 1}=0$.

It is proven in the same way that $R_{, 2}=0$. The remainder of the proof is obvious.

LEMMA A.2. Let $v^{\epsilon} \in \mathcal{C}^{\epsilon}$ be such that $\mathcal{O}\left(v^{\epsilon}\right)=0$, then $-2 \leq \mathcal{O}\left(E^{\epsilon}\right) \leq 1$. Moreover, the order of the strains and the associated displacements are such that

(i) $\mathcal{O}\left(E^{\epsilon}\right)=-2$ if and only if $v^{\epsilon}$ is not of Class $\mathbf{0}$,

(ii) $\mathcal{O}\left(E^{\epsilon}\right) \geq 0$ if and only if $v^{\epsilon}$ is of Class $\mathbf{0}$,

(iii) $\mathcal{O}\left(E^{\epsilon}\right)=1$ if and only if $v_{-}$is of Class 1,

Proof. Since $\mathcal{O}\left(v^{\epsilon}\right)=0$, we know from lemma 3.2 that the only non-zero components of the strain development $E^{-2}$ are $E_{\alpha \beta}^{-2}(v)=\frac{1}{2} v_{, \alpha}^{0} \cdot v_{, \beta}^{0}$ which gives (i).

If $v^{\epsilon}$ is of Class $\mathbf{0}$ then $E^{-2}=E^{-1}=0$ which implies (ii). Moreover,

$$
\begin{aligned}
& 2 E_{\alpha \beta}^{0}=\left(v_{, \alpha}^{1}+e_{\alpha}\right) \cdot\left(v_{, \beta}^{1}+e_{\beta}\right)-\delta_{\alpha \beta}, \\
& 2 E_{\alpha 3}^{0}=\left(v_{, \alpha}^{1}+e_{\alpha}\right) \cdot\left(V^{\prime}+e_{3}\right), \quad 2 E_{33}^{0}=\left(\left\|V^{\prime}+e_{3}\right\|^{2}-1\right) .
\end{aligned}
$$

Therefore, $E^{0}=0$ if and only if the matrix $\left(e_{1}+v_{, 1}^{1}(x)\left|e_{2}+v_{, 2}^{1}(x)\right| e_{3}+V^{\prime}\left(x_{3}\right)\right)$ is an orthogonal matrix, say $R(x)$. Since $v^{\epsilon} \in \mathcal{C}^{\epsilon}, R(x) \in S O(3)$. We can then use Lemma A. 1 and conclude that $v^{\epsilon}$ is of Class 1 .

Conversely let us consider $v^{\epsilon}$ of Class $\mathbf{1}$. Then $E^{p}=0$ for $p \leq 0$. To complete the proof, it remains to establish that $E^{1} \neq 0$. Its component $E_{33}^{1}$ reads as

$$
E_{33}^{1}=v_{, 3}^{1} \cdot\left(V^{\prime}+e_{3}\right)=\left(\bar{V}^{\prime}+x_{\alpha} R^{\prime} e_{\alpha}\right) \cdot R e_{3} .
$$

So that it vanishes, it is necessary that $R^{\prime} e_{\alpha} \cdot R e_{3}=0$. But since $R\left(x_{3}\right) \in S O(3)$ we have also $R^{\prime} e_{\alpha} \cdot R e_{3}+R^{\prime} e_{3} \cdot R e_{\alpha}=0$ and $R^{\prime} e_{3} \cdot R e_{3}=0$. Then we should have $R^{\prime} e_{3}=V^{\prime \prime}=0$ and hence, by virtue of the boundary condition (see Remark 4.3), $V=0$. This is impossible, because $\mathcal{O}\left(v^{\epsilon}\right)=0$. So $E_{33}^{1} \neq 0$ and the proof is complete.

LEMMA A.3. Let $v^{\epsilon} \in \mathcal{C}^{\epsilon}$ be such that $\mathcal{O}\left(v^{\epsilon}\right)=1$, then $0 \leq \mathcal{O}\left(E^{\epsilon}\right) \leq 2$. Moreover, the order of the strains and the associated displacements are such that

(1) $\mathcal{O}\left(E^{\epsilon}\right) \geq 1$, if and only if $v^{\epsilon}$ is of Class 2,

(2) $\mathcal{O}\left(E^{\epsilon}\right)=2$, if and only if $v^{\epsilon}$ is of Class 3 with $q=1$. 
Proof. Let $v^{\epsilon} \in \mathcal{C}^{\epsilon}$ be such that $\mathcal{O}\left(v^{\epsilon}\right)=1$, recalling lemma 3.2, we immediately see that $E^{-2}=E^{-1}=0$ and $E_{33}^{0}=0,2 E_{\alpha 3}^{0}=v_{, \alpha}^{1} \cdot e_{3}, 2 E_{\alpha \beta}^{0}=\left(v_{, \alpha}^{1}+\right.$ $\left.e_{\alpha}\right) \cdot\left(v_{, \beta}^{1}+e_{\beta}\right)-\delta_{\alpha \beta}$. Therefore, $E^{0}=0$ if and only if the matrix $R(x)=\left(v_{1}^{1}(x)+\right.$ $\left.e_{1}\left|v_{, 2}^{1}(x)+e_{2}\right| e_{3}\right)$ is a rotation. Because of Lemma A.1, $R(x)$ depends only on $x_{3}$ and, since $R\left(x_{3}\right) e_{3}=e_{3}$, we easily deduce that $v^{\epsilon}$ is of Class 2 and so (i).

Let us prove (ii). When $v^{\epsilon}$ is of Class 2, a direct calculation gives

$$
\begin{aligned}
& 2 E_{\alpha \beta}^{1}=R\left(x_{3}\right) e_{\alpha} \cdot v_{, \beta}^{2}+R\left(x_{3}\right) e_{\beta} \cdot v_{, \alpha}^{2}, \quad E_{33}^{1}=V_{3}^{\prime}\left(x_{3}\right), \\
& 2 E_{\alpha 3}^{1}=x_{\beta} R\left(x_{3}\right) e_{\alpha} \cdot R^{\prime}\left(x_{3}\right) e_{\beta}+R\left(x_{3}\right) e_{\alpha} \cdot V^{\prime}\left(x_{3}\right)+v_{3, \alpha}^{2} .
\end{aligned}
$$

Let us first assume that $E^{1}=0$. From $E_{33}^{1}=0$ and the boundary condition, we deduce that $V_{3}=0$. The conditions $E_{\alpha 3}^{1}=0$ reads as

$$
\begin{aligned}
& 0=x_{\beta} R^{\prime} e_{\beta} \cdot R e_{1}+V^{\prime} \cdot R e_{1}+v_{3,1}^{2} \\
& 0=x_{\beta} R^{\prime} e_{\beta} \cdot R e_{2}+V^{\prime} \cdot R e_{2}+v_{3,2}^{2} .
\end{aligned}
$$

Differentiating (A.2) with respect to $x_{2}$ and (A.3) with respect to $x_{1}$ leads to $R^{\prime} e_{1} \cdot R e_{2}=R^{\prime} e_{2} \cdot R e_{1}$. But, since $R\left(x_{3}\right) \in S O(3)$ we have also $R^{\prime} e_{1} \cdot R e_{2}+R^{\prime} e_{2} . R e_{1}$ $=0$. So $R^{\prime} e_{1} \cdot R e_{2}=0$. We deduce also from $R e_{1} \cdot R e_{1}=1$ that $R^{\prime} e_{1} \cdot R e_{1}=0$, and, from $R e_{1} \cdot R e_{3}=R e_{1} \cdot e_{3}=0$ that $R^{\prime} e_{1} \cdot R e_{3}=0$. Consequently $R^{\prime} e_{1}=0$. It is proved in the same way that $R^{\prime} e_{2}=0$ and, since $R e_{3}=e_{3}$, we finally obtain $R^{\prime}=$ 0 . So, thanks to the boundary condition, $R=I$. Inserting these results into the conditions $E_{\alpha \beta}^{1}=E_{\alpha \beta}^{1}=0$ leads to

$$
v_{\beta, \alpha}^{2}+v_{\alpha, \beta}^{2}=0, \quad v_{3, \alpha}^{2}=-V_{\alpha}^{\prime},
$$

from which we deduce that $v^{\epsilon}$ is of Class 3 .

Let us now assume that $v^{\epsilon}$ is of Class 3 with $\mathcal{O}\left(v^{\epsilon}\right)=1$. Then one first verifies that $E^{p}=0$ when $p \leq 1$. It remains to prove that $E^{2} \neq 0$. Let us consider $E_{33}^{2}$, a direct calculation gives

$$
E_{33}^{2}=\frac{1}{2} V^{\prime} \cdot V^{\prime}+\bar{V}_{3}^{\prime}-x_{\alpha} V_{\alpha}^{\prime \prime}
$$

If $E_{33}^{2}=0$, then $V^{\prime \prime}=0$ and, owing to the boundary condition, $V=0$. But, since $\mathcal{O}\left(v^{\epsilon}\right)=1$, it is not possible, so $E_{33}^{2} \neq 0$.

LEMMA A.4. Let $v^{\epsilon} \in \mathcal{C}^{\epsilon}$ be such that $\mathcal{O}\left(v^{\epsilon}\right)=q$, with $q \leq 2$, then $q-1 \leq$ $\mathcal{O}\left(E^{\epsilon}\right) \leq q+1$. Moreover,

(i) $\mathcal{O}\left(E^{\epsilon}\right) \geq q$, if and only if $v^{\epsilon}$ is of Class 4 ,

(ii) $\mathcal{O}\left(E^{\epsilon}\right)=q+1$, if and only if $v^{\epsilon}$ is of Class 3 ,

Proof. Let $v^{\epsilon} \in \mathcal{C}^{\epsilon}$ be such that $\mathcal{O}\left(v^{\epsilon}\right)=q \geq 2$. Then $E^{p}=0$ for $p \leq q-2$ and $E^{q-1}$ reads as

$$
2 E_{\alpha \beta}^{q-1}=v_{\beta, \alpha}^{q}+v_{\alpha, \beta}^{q}, \quad 2 E_{\alpha 3}^{q-1}=v_{3, \alpha}^{q}, \quad E_{33}^{q-1}=0 .
$$


From (A.4) we deduce that $E^{q-1}=0$ if and only if

$$
v^{q}(x)=w\left(x_{3}\right) e_{3 \wedge} x+V\left(x_{3}\right),
$$

hence (i) by definition of Class 4 .

One considers from now on only $v^{\epsilon}$ of Class 4 . To prove (ii) we must distinguish the case $q=2$ from the case $q>2$.

Let us first consider the case $q=2$. Then $E^{2}$ reads as

$$
2 E_{\alpha \beta}^{2}=w^{2} \delta_{\alpha \beta}+v_{\beta, \alpha}^{3}+v_{\alpha, \beta}^{3}, \quad 2 E_{\alpha 3}^{2}=w^{\prime} e_{\alpha} \cdot\left(e_{3 \wedge} x\right)+V_{\alpha}^{\prime}+v_{3, \alpha}^{3}, \quad E_{33}^{2}=V_{3}^{\prime} .
$$

Let us assume that $E^{2}=0$. From $E_{33}^{2}=0$ and the boundary condition, we deduce that $V_{3}=0$. The conditions $E_{\alpha 3}^{2}=0$ reads as

$$
\begin{aligned}
& 0=-w^{\prime} x_{2}+V^{\prime}{ }_{1}+v_{3,1}^{3} \\
& 0=-w^{\prime} x_{1}+V_{2}^{\prime}+v_{3,2}^{3} .
\end{aligned}
$$

Differentiating (A.6) with respect to $x_{2}$ and (A.7) with respect to $x_{1}$ leads to $w^{\prime}=0$. Thus, thanks to the boundary condition, $w=0$. Inserting this property in the conditions $E_{\alpha \beta}^{2}=E_{\alpha 3}^{2}=0$ leads to

$$
v_{\beta, \alpha}^{3}+v_{\alpha, \beta}^{3}=0, \quad v_{3, \alpha}^{3}=-V_{\alpha}^{\prime},
$$

from which we deduce easily that $v^{\epsilon}$ is of Class 3.

Conversely, if $v^{\epsilon}$ is of Class 3 with $\mathcal{O}\left(v^{\epsilon}\right)=2$, then $E^{p}=0$ for $p \leq 2$. Moreover $E_{33}^{3}=-x_{\alpha} V^{\prime \prime}{ }_{\alpha}+\bar{V}^{\prime}$. Consequently, if $E_{33}^{3}=0$, we should have $V^{\prime \prime}=0$ and hence $V=0$, what is impossible since $\mathcal{O}\left(v^{\epsilon}\right)=2$. We have proved (ii) when $q=2$.

Let us now consider the case $q>2$. Then $E^{q}$ reads as

$$
2 E_{\alpha \beta}^{q}=v_{\beta, \alpha}^{q+1}+v_{\alpha, \beta}^{q+1}, \quad 2 E_{\alpha, \beta}^{q}=w^{\prime} e_{\alpha} \cdot\left(e_{3 \wedge} x\right)+V_{\alpha}^{\prime}+v_{3, \alpha}^{q+1}, \quad E_{33}^{q}=V_{3}^{\prime} .
$$

Only $E_{\alpha \beta}^{q}$ differs, the term $w^{2}$ having disappeared. Since $E_{\alpha 3}^{q}$ and $E_{33}^{q}$ remain unchanged, we can proceed as is the case $q=2$. The proof of the Lemma is complete.

\section{References}

1. E. Acerbi, G. Buttazzo and D. Percivale, A variational definition for the strain energy of an elastic string. J. Elast. 25 (1991) 137-148.

2. S.S. Antman, The Theory of Rods, Vol. VIa/2 of Handbuch der Physik. Springer, Berlin Heidelberg New York (1972), pp. 641-703.

3. S.S. Antman, Non-linear Problems of Elasticity, Vol. 107 of Applied Mathematical Sciences. Springer, Berlin Heidelberg New York (1995). 
4. J.M. Ball, Convexity conditions and existence theorems in nonlinear elasticity. Arch. Ration. Mech. Anal. 63 (1976) 337-403.

5. J.M. Ball and F. Murat, $W^{1, p}$-quasiconvexity and variational problems for multiple integrals. J. Funct. Anal. 58 (1984) 225-253.

6. A. Bermúdez and J.M. Viaño, Une justification des équations de la thermoélasticité des poutres à section variable par des méthodes asymptotiques. RAIRO Anal. Numér. 18 (1984) 347-376.

7. P.G. Ciarlet, Mathematical Elasticity, Vol. I. North-Holland, Amsterdam (1988).

8. P.G. Ciarlet and P. Destuynder, A justification of a nonlinear model in plate theory. Comput. Methods Appl. Mech. Eng. 17/18 (1979) 227-258.

9. A. Cimetière, G. Geymonat, H. Le Dret, A. Raoult and Z. Tutek, Asymptotic theory and analysis for displacements and stress distribution in nonlinear elastic straight slender rods. J. Elast. 19 (1988) 111-161.

10. B. Dacorogna, Direct Methods in the Calculus of Variations. Applied Mathematical Sciences 78, Springer-Verlag (1989).

11. B. Dacorogna and P. Marcellini, Existence of minimizers for non-quasiconvex integrals. Arch. Ration. Mech. Anal. 131 (1995) 359-399.

12. D.D. Fox, A. Raoult and J.C. Simo, A justification of nonlinear properly invariant plate theories. Arch. Ration. Mech. Anal. 124 (1993) 157-199.

13. G. Friesecke, R.D. James and S. Müller, A hierarchy of plate models derived from nonlinear elasticity by $\Gamma$-convergence. (2005) Preprint.

14. G. Geymonat, F. Krasucki and J.-J. Marigo, Stress distribution in anisotropic elastic composite beams. In: P. Ciarlet and E. Sanchez-Palencia (eds.), Applications of Multiple Scalings in Mechanics, Masson (1987), pp. 118-133.

15. G. Geymonat, F. Krasucki and J.-J. Marigo, Sur la commutativité des passages à la limite en théorie asymptotique des poutres composites. C. R. Acad. Sci. Sér. 2305 (1987) 225-228.

16. M.E. Gurtin, The linear theory of elasticity, Vol. VIa of Handbuch der Physik. Springer, Berlin Heidelberg New York (1972), pp. 1-296.

17. R. Jamal and E. Sanchez-Palencia, Théorie asymptotique des tiges courbes anisotropes. C. $R$. Acad. Sci. Sér. 1322 (1996) 1099-1106.

18. H. Le Dret and A. Raoult, The nonlinear membrane model as variational limit of nonlinear three-dimensional elasticity. J. Math. Pures Appl. 75 (1995) 551-580.

19. H. Le Dret and A. Raoult, The quasiconvex envelope of the Saint Venant-Kirchhoff stored energy function. Proc. R. Soc. Edin., A 125 (1995) 1179-1192.

20. A.E.H. Love, A Treatise on the Mathematical Theory of Elasticity, 4th edn. Dover, New York (1927).

21. J.-J. Marigo, H. Ghidouche and Z. Sedkaoui, Des poutres flexibles aux fils extensibles: une hiérarchie de modèles asymptotiques. C. R. Acad. Sci. Paris 326(Série IIb) (1998) 79-84.

22. J.-J. Marigo and K. Madani, The influence of the type of loading on the asymptotic behavior of slender elastic rings. J. Elast. 75 (2004) 91-124.

23. N. Meunier, Modélisation de fils en élasticité tridimensionnelle. Thèse, Université Pierre et Marie Curie, Paris (2003).

24. N. Meunier, Recursive derivation of one-dimensional models from three-dimensional nonlinear elasticity. Math. Mech. Solids. In press.

25. M.G. Mora and S. Müller, Derivation of the nonlinear bending-torsion theory for inextensible rods by Gamma-convergence. Calc. Var. 18 (2002) 287-305.

26. M.G. Mora and S. Müller, A nonlinear model for inextensible rods as a low energy $\Gamma$-limit of three-dimensional nonlinear elasticity. Ann. Inst. Henri Poincaré, Anal. nonlinéaire 21 (2004) 271-299.

27. C.B. Morrey, Quasi-convexity and the lower semicontinuity of multiple integrals. Pac. J. Math. 2 (1952) 25-53. 
28. F. Murat and A. Sili, Comportement asymptotique des solutions du système de l'élasticité linéarisée anisotrope hétérogéne dans des cylindres minces. C. R. Acad. Sci., Ser. 1328 (1999) 179-184.

29. O. Pantz, Quelques problèmes de modélisation en élasticité non linéaires. Thèse, Univer. P. et M. Curie, Paris (2001).

30. A. Raoult, Nonpolyconvexity of the stored energy function of a Saint-Venant Kirchhoff material. English. Russian, Czech summary, 31 (1986) 417-419.

31. A. Rigolot, Sur une théorie asymptotique des poutres. J. Méc. 11(2) (1972) 673-703.

32. J. Sanchez-Hubert and E. Sanchez-Palencia, Couplage flexion torsion traction dans les poutres anisotropes à sections hétérogènes. C. R. Acad. Sci. Paris 312 (1991) 337-344.

33. J. Sanchez-Hubert and E. Sanchez-Palencia, Statics of curved rods on account of torsion and flexion. Eur. J. Mech. A, Solids 18 (1999) 365-390.

34. S.P. Timoshenko, Strength of Materials, Parts 1 and 2, 3rd edn. Krieger (1983).

35. L. Trabucho and J.M. Viaño, Mathematical modelling of rods. In: P.G. Ciarlet and J.-L. Lions (eds.), Handbook of Numerical Analysis, Vol. IV. Norh-Holland, Amsterdam (1996). 